\title{
Sun-Synchronous Planetary Exploration
}

\author{
William "Red" Whittaker, George Kantor, Ben Shamah, David Wettergreen \\ email: $\{$ red, kantor, bshamah, dsw \} @ri.cmu.edu \\ Field Robotics Center, The Robotics Institute \\ Carnegie Mellon University \\ Pittsburgh PA 15213 \\ Phone: (412) 268-6559; Fax: (412) 268-5895
}

\begin{abstract}
Contemporary planetary missions are characterized by favorable landing sites, communication, and duration. The ambition of planetary exploration is to discover the unknown: to explore regions, not just sites; to analyze, not just observe; and to operate effectively and reliably without excessive communication and ground support. New robotics technologies and mission concepts will revolutionize exploration systems and operations. This paper addresses the key enabling technologies for sun-synchronous planetary exploration: environmental modeling, temporal path planning, scaling, and robot configuration.
\end{abstract}

\section{INTRODUCTION}

Sun-synchronous navigation is accomplished by moving opposite the rotation of a planet to remain continually in sunlight. This enables the robotic capability of persistent, in some cases perpetual, presence to explore, dwell in, and develop resource-rich regions near the poles of planets and moons. This also enables unprecedented range of operation. By clocking with the sun, a planetary rover will maintain continual exposure to sunlight as it travels, with advantages to survivability, range and persistent power.

By lagging the night-to-day terminator by the appropriate phase angle, a rover can regulate moderate temperature or seek transients between cold and hot.

The ability of rovers to dodge shadows, seek sunlight, and drive sun-synchronous routes could lay the foundation for new, imaginative missions and enterprises on moons and planets. These missions will deepen scientific inquiry, extend our reach in space, develop and extract in situ resources, and enable human visits to other planets.

\subsection{Motivation}

Because solar radiation is the power source of choice for exploration of the inner solar system, the availability of prolonged sunlight in polar regions makes sun-synchronous operation a promising scenario for long-term presence. Through temporal path planning, sun-synchronous rovers can schedule the collection of solar power, so that power storage requirements are minimized. This reduced need translates into reduced rover weight, complexity, and cost.

Figure 1. Sun-Synchronous Rover Concept

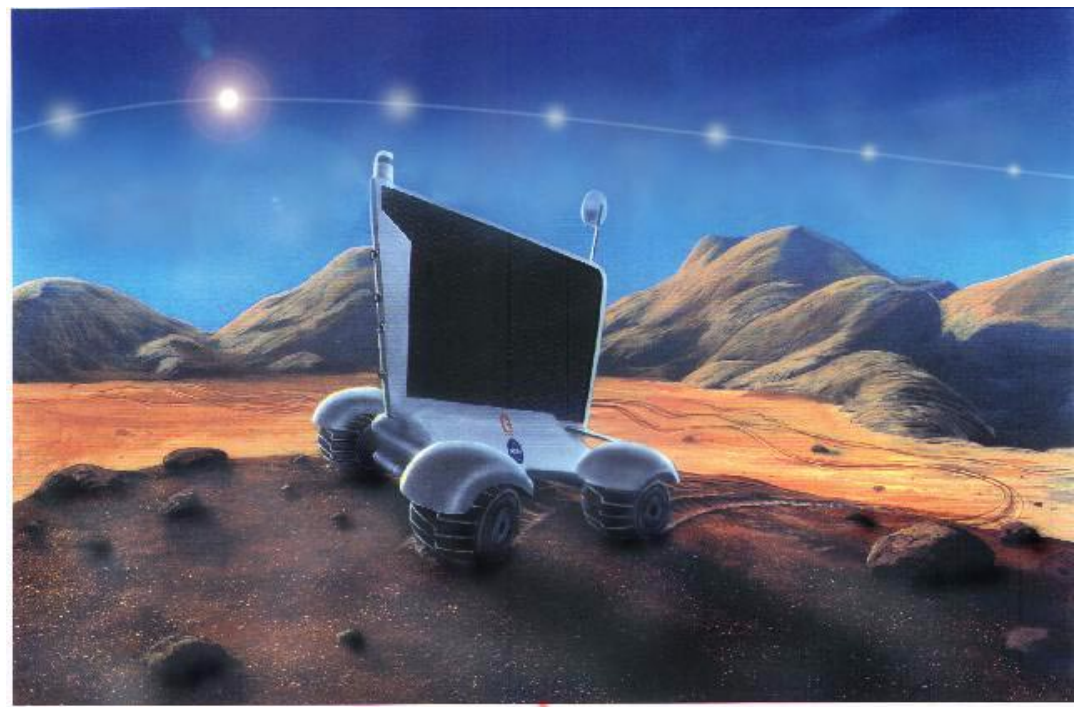


Sun-synchrony enables coverage of vast regions far from a landing site. This model of robotic operations allows diverse and detailed exploration that is not possible with traditional approaches. At mid-latitudes this sun synchrony results in global circumnavigation while at high-latitudes, during periods of persistent sunlight, the sun synchronous route is a circumfeature circuit each local day.

By their identification and mapping of sun routes, sunsynchronous rovers will enable the installation of permanent power collection infrastructure in polar regions. Solar arrays could be distributed in a ring along the route so that some arrays are exposed to sunlight at all times. When connected by conductors, the resulting ring would provide a continual source of power for manufacturing facilities and scientific outposts such as telescope arrays and accommodations for human visits.

A tantalizing feature of polar regions of moons and planets is the possible existence of volatiles such as water ice. The Lunar Prospector mission found evidence of hydrogen in permanently dark craters near the poles of the Moon [Feldman et al, 1998]. Radar data suggests that water may exist on Mercury [Ingersoll et al, 1992].

The discovery, extraction, and processing of polar volatiles will require an extended presence made possible by sun-synchronous navigation capabilities. During its trek, a sun-synchronous rover will have opportunities to make short forays into permanently dark craters to look for and extract volatiles. Mining water from permanently dark craters to reduce a habitat's dependence on fully recyclable life support systems is one of many scenarios for production from in situ resources.

Other investigations, such as origins, deep impacts, or time-history of axis tilt, are unique polar agendas.

On Mercury and the Moon, moving bands of moderate temperature exist on the sun side of the night-to-day terminator. Polar regions of the Moon experience temperatures in the range of $220 \mathrm{~K}+/-$ only $10 \mathrm{~K}$ with seasonal fluctuation [Heiken et al, 1991]. This contrasts dramatically with equatorial regions with temperatures of $254 \mathrm{~K}+/-140 \mathrm{~K}$ that swing from one extreme to the other every lunar day.

By pacing its movement through one of these moderate bands, a sun-synchronous rover will choose its operating temperature, possibly moving and working in a temperature range comparable to room temperature on Earth. This scenario precludes the challenge of repeated day/night cycles of scorching heat and cryogenic cold found in equatorial regions and eliminates the need for massive rover designs, hibernations, complicated mission protocols, and nuclear heating measures. Sun-synchronous rovers may not require active thermal protection thus minimizing mass and cost.

Sun-synchronous circumnavigation will make possible an innovative class of planetary rovers notable for their lower mass, reduced cost, and elimination of reliance on nuclear power or night heating. This powerful capability will engender robotic rovers that are free to travel, explore and work at will across vast expanses. Combining longevity with range, sun-synchronous rovers will pave the way for space endeavors that further science, exploration, in
Figure 2. Temperature bands and the night-to-day terminator

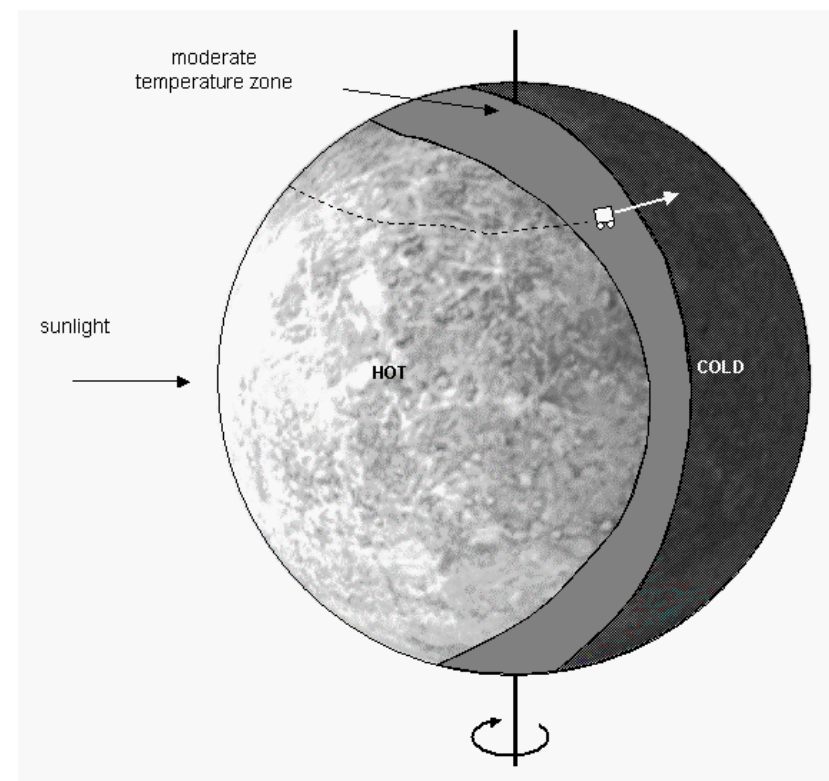

situ resource extraction, and self-sufficient human presence.

\section{TECHNOLOGIES}

This paper addresses four key enabling technologies for sun-synchronous planetary exploration: environmental modeling, temporal path planning, scaling, and robot configuration.

\subsection{ENVIRONMENTAL MODELING}

A clear understanding of the variable relationship between environment and the robot is needed for evaluating sun-synchronous routes and enabling sun-synchronous exploration. The motion of the sun at a particular location combined with terrain elevation models indicate whether the sun is visible at a given location, and at which angle the sunlight will be incident on solar panels. The geometry given by ephemeris data can be used to generate the sunlight model as a function of surface topography. Rover dynamics and terramechanic models can be used to predict the power consumption of a rover traversing the landscape. Together with models of lighting and terrain dynamics estimates of the ratio of available power (sunlight) to required power can be generated. This combination is crucial to the capability of determining successful rover trajectories.

\subsection{TEMPORAL PATH PLANNING}

Rough mission routes can be planned a priori from orbital mapping. These paths must be both temporal and spatial. In order to optimize solar power gain each waypoint is only good for a specific duration of time due to the changing position of the Sun. On a perfectly spherical planet an arctic circle route might constitute a mission path for global circumnavigation. In planetary terms, mission paths will take the form of a sequence of points and regions 
such that movement from region to region accomplishes the circumnavigation. Path planners must select these regions to be large and widely spaced so that the rover has sufficient latitude to reach subgoals in the presence of unknown obstructions.

Since planetary bodies are not perfect spheres a second concern of the path planner is to ensure that local terrain features do not combine with the position of the Sun to create shadowed regions in which the rover cannot derive power. Using its environmental model, the rover must solve this combined spatial and temporal problem so that it can dodge shadows, remain sun-synchronous, and progress along a continuously lit path. (See Figure 3, top.)

Below the arctic latitudes this sun synchrony results in global circumnavigation. While at the equator on most planetary bodies this requires excessive speed, at high latitudes the path plan moves the vehicle at a pace that allows dense exploration. For example on the Moon at $80^{\circ}, 3 \mathrm{~km} /$ $\mathrm{hr}$ average progress, below typical walking speed, is all that is required to maintain sun synchrony. (See Figure 3, bottom.)

Above the arctic latitude in which perpetual sunlight exists for part (or all) of the year a sun-synchronous rover can make circular excursions with period equal to the length of the local day.

Circumfeature operation denotes the capability of a rover to continually circumnavigate a local feature(s). With an articulated solar array, requirements on path are relaxed further as the vehicles direction of travel can deviate further from the motion of the Sun. The possibility of sunsynchronous operation near planetary poles provides a viable means of thorough exploration and long-duration operation.

\subsection{SCALING}

Circumnavigation properties can be scaled and generalized from planet to planet depending on the planet's characteristics. Advantageous planet attributes for sun-synchronous exploration include: slow rotation, small radius, low gravity, closer sun proximity, and small axial tilt. In this way, one can imagine classes of traverses that are "equivalent" in some respect. An example would be "rate equivalence" of the set of sun tracking traverses that can be completed at a given rate. A traverse with a circumference of 24 kilometers around an arctic feature on Earth must execute in 24 hours, resulting in a required average speed of $1 \mathrm{~km} /$ hour. With a 4,224 hour day, a rate equivalent trek on Mercury would constitute a 4,224 km circumnavigation of the planet at 74 degrees latitude. Other analogs can be drawn for power consumption due to soil resistance (which scales with gravity), power collection (which scales with solar irradiance), and minimum circumnavigation route for perpetual light (a function of axial tilt).

Equatorial circumnavigation of a planet would require a rover to travel at: $1670 \mathrm{~km} / \mathrm{hr}$ on Earth, $15 \mathrm{~km} / \mathrm{hr}$ on the Moon, and $4 \mathrm{~km} / \mathrm{hr}$ on Mercury. Mercury has the benefit of one third the gravity of earth and 7 times the solar flux which translates to a mobility power advantage of 20 .
Three possible sun-synchronous traverses for the Earth, Moon, and Mercury are depicted in Table 1. For each case the average speed, average power, solar array size, and trek length are calculated. The required average speed is calculated from the trek length and the diurnal period of the Sun. The power required is an idealized calculation shown in equation 1. The constant of 50 Watts is an assumed value that includes the power for all systems except the locomotion of the rover (e.g. computing, communication, ...) . The simplified equation presented here is merely to highlight the effects of planet gravity and required speed on required power. Effects of varied: terrain, thermal conditions, communication requirements, computation and sensing requirements are not taken into account.

\subsection{ROBOT CONFIGURATION}

Sun-synchronous rovers will collect solar power in a manner distinct from equatorial counterparts. The common view of a planetary rover has a solar array deployed as a

Figure 3. Circumpolar and Circumfeature Paths on the Lunar South Pole
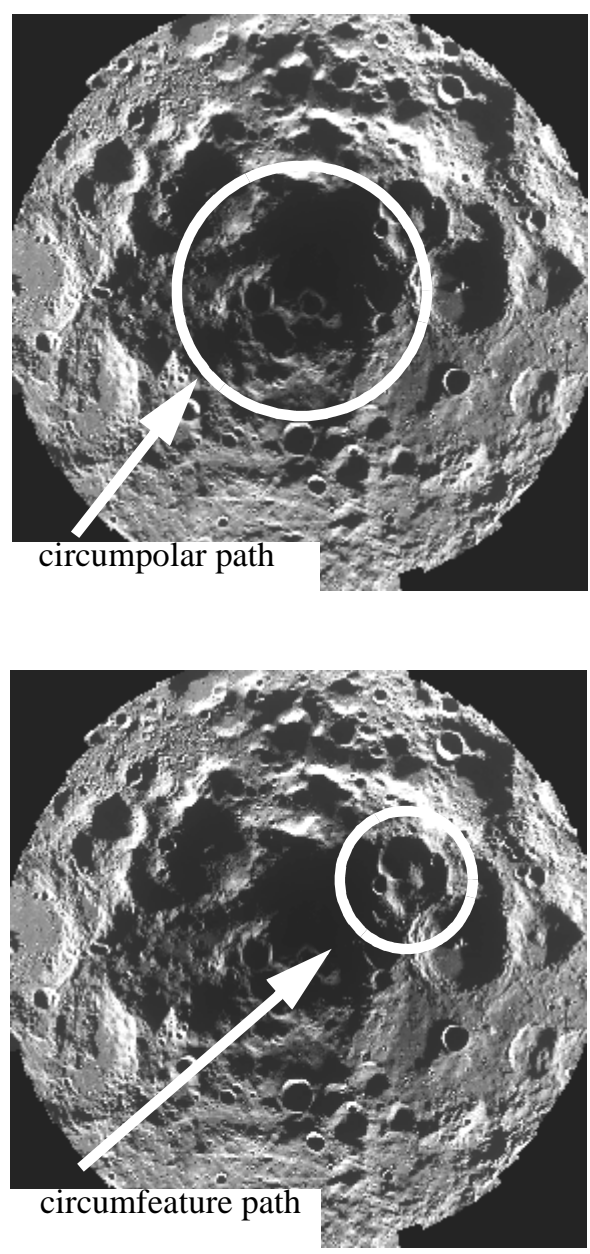
Table 1: Sun-Synchronous Traverse Data

\begin{tabular}{|c|c|c|c|c|c|}
\hline & & Units & Earth & Moon & Mercury \\
\hline \multirow[t]{4}{*}{$\overline{\text { Equatorial }}$} & Required average speed & km/hour & $\overline{1670}$ & $\overline{15}$ & $\overline{4}$ \\
\hline & $\begin{array}{l}\text { Required power } \\
(100 \mathrm{~kg} \text { rover, soil resistance } 0.1)\end{array}$ & Watts & 45412 & 119 & 78 \\
\hline & $\begin{array}{l}\text { Required solar array size } \\
(20 \% \text { efficiency })\end{array}$ & $\mathrm{m}^{2}$ & 227 & 0.4 & 0.04 \\
\hline & Trek length & $\mathrm{km}$ & 40074 & 10914 & 15331 \\
\hline \multirow{4}{*}{ Polar, 80 degrees latitude } & Required average speed & $\mathrm{km} /$ hour & 290 & 3 & 0.6 \\
\hline & $\begin{array}{l}\text { Required power } \\
(100 \mathrm{~kg} \text { rover, soil resistance } 0.1)\end{array}$ & Watts & 7927 & 62 & 55 \\
\hline & $\begin{array}{l}\text { Required solar array size } \\
(20 \% \text { efficiency) }\end{array}$ & $\mathrm{m}^{2}$ & 40 & 0.23 & 0.03 \\
\hline & Trek length & $\mathrm{km}$ & 6959 & 1895 & 2662 \\
\hline \multirow[t]{4}{*}{5 kilometer radius feature } & Required average speed & $\mathrm{km} / \mathrm{hour}$ & 1 & 0.04 & 0.01 \\
\hline & $\begin{array}{l}\text { Required power } \\
(100 \mathrm{~kg} \text { rover, soil resistance } 0.1)\end{array}$ & Watts & 86 & 50.2 & 50.1 \\
\hline & $\begin{array}{l}\text { Required solar array size } \\
(20 \% \text { efficiency })\end{array}$ & $\mathrm{m}^{2}$ & 0.4 & 0.18 & 0.03 \\
\hline & Trek length & $\mathrm{km}$ & 31 & 31 & 31 \\
\hline \multirow[t]{5}{*}{ Parameters } & Diurnal period & Earth Days & 1 & 30 & 176 \\
\hline & Planet radius & $\mathrm{km}$ & 6378 & 1737 & 2440 \\
\hline & Axial tilt & degrees & 23 & 2 & 0.01 \\
\hline & Surface gravity & $\mathrm{m} / \mathrm{s}^{2}$ & 9.8 & 1.6 & 2.8 \\
\hline & Solar irradiance & $\mathrm{W} / \mathrm{m}^{2}$ & 1000 & 1368 & 9127 \\
\hline
\end{tabular}

Equations:

power required $=($ rover mass $)($ gravity $)($ velocity $)($ soil resistance $)+$ constant

constant $=50$ Watts for all cases

solar panel area $=\frac{\text { power required }}{(\text { solar irradiance })(\text { solar panel efficiency })}$

level deck, facing an overhead sun (like the Rocky, Sojourner, and Fido rovers). Sun-synchronous rovers will need vertical solar arrays orthogonal to the sunlight which comes from low on the horizon. If such an array is fixed, power insolation is directly coupled to vehicle steering, creating addition constraints to sun synchronous navigation planning. A steerable vertical array decouples power insolation planning from rover steering at the cost of actuation, displaced volume due to swept motion, and thermal consequences.

\section{EVOLUTIONARY PRESENCE}

With a unique concurrence of features such as moderate temperatures, extended periods of sunlight, and the possibility of in situ volatiles, polar regions of moons and planets offer excellent opportunities for long term exploratory missions, manufacturing of resources, and venues for human visits. Sun-synchronous presence will pave the way for future space endeavors including scientific inquiry, exploration, installation of solar power grids, in situ resource extraction, and human operations.

Sun-synchronous operation should not be thought of as an end in itself. Rather, it should be thought of as a means to an end; a toe hold from which a sustained presence can incrementally be established on remote planetary surfaces. This section outlines a vision for this evolutionary presence which is enabled by sun-synchronous technology.

\subsection{REGIONAL EXPLORATION}

Once a sun-synchronous route has been established it can be used as a foundation for a more thorough exploration of the region containing the sun-synchronous route. A rover traversing an established route can explore the surrounding region by deviating slightly from the route, always allowing a margin of safety that allows it to return to the established route while maintaining sun synchrony. Each time the rover deviates from the original route, it establishes new routes which can be used in future itera- 
tions. In this way, the sun-synchronous route is incrementally expanded into a network of routes to provide coverage over a given region.

\subsection{ESTABLISHMENT OF SOLAR GRIDS AND PERMANENT INFRASTRUCTURE}

By the definition of a sun-synchronous route, solar arrays can be distributed along the route so that some arrays are exposed to sunlight at all times. When connected by conductors, the resulting ring would provide a continual source of solar power.

The existence of such a solar power grid makes it possible to maintain an extended surface presence without having to continually move with the sun. Rovers without sun-synchronous capability will be able to operate on the route, tapping into the power grid when necessary. In a similar manner, stationary structures established along a sun-synchronous route can be provided continual power. Scientific outposts, manufacturing facilities, and human dwelling space can all be established in this manner.

\subsection{PERMADARK EXPLORATION}

Volatiles such as water that are suspected to reside in the bottoms of permanently dark craters near the poles of Mercury and The Moon. The existence of in situ water has obvious ramifications for the prospect of human presence; water will be essential to the sustenance of human life. Mining water from permadark craters would enable the production of drinking water and oxygen, reducing a habitat's dependence on fully recyclable life support centers. Rovers capable of entering permadark cold traps will be necessary to find and extract these valuable volatile resources.

Transient missions by sun-synchronous rovers provide one method of entering and returning from a permanently dark crater. Using this technique, a sun-synchronous rover simply enters the darkness, relying on-board batteries and its own thermal mass to survive the cold. A rover operating in this mode would have to manage its on-board resources and choose a route which will allow it to return to safety within the allotted time. Such a trip would be short by necessity, but would provide an important first step into permadark exploration.

A second way to enter a permadark crater is made possible by power beaming. In this scenario, one rover remains in sunlight at the rim of the crater. A second rover enters the crater, maintaining continual line-of-sight with the rover on the rim. The first rover transmits power to the rover in the crater via a laser beam or microwaves. This technique will allow for extended permadark missions which could be used to extract volatiles.

\subsection{OUTPOSTS FOR DEEP SPACE EXPLORATION}

As outlined in the previous sections, sun-synchronous operation will make possible permanent infrastructure including manufacturing facilities and human habitat. Such an outpost established on The Moon could serve as a launching point for deep space missions. Spacecraft manufactured in lunar orbit could be propelled into deep space with a fraction of the $\Delta v$ required to launch a craft from Earth orbit. The spacecraft components could be fabricated at the surface outpost using primarily in situ resources. The components would then be launched into lunar orbit and assembled in space. An operation of this form would greatly reduce the cost of deep space missions and extend the reaches of humanity into the outer solar system and beyond.

\section{CONCLUSION}

This paper has described the concept of sun-synchronous navigation. We have discussed immediate ramifications of sun-synchronous capability to planetary surface missions, and we have outlined a vision of the long term impact the concept will have on the future of space exploration.

Are there circumpolar sun-synchronous routes? That remains an unknown until a robot circles a pole and traces back on its own tracks. Earth explorers like Lewis and Clark failed in their pursuit of a Northwest passage, but discovered immense knowledge of our unexplored West in their failed attempt. Magellan settled the question of an oceanic circumnavigation route by successfully encircling the Earth. Planetary Magellan missions could discover surface routes, assay volatiles, transform our view of the solar system, and drive robotic technologies to further explore the high frontier.

\section{REFERENCES}

[Beatty et al, 1990] J.K. Beatty and A. Chaikin, eds., The New Solar System (Third Edition), Sky Publishing Corp. and Cambridge University Press, 1990

[Bussey et al, 1999] D. B. J Bussey, M. S. Robinson, P. D. Spudis, "Illumination Conditions at the Lunar Poles," in Lunar and Planetary Science XXX, abstract \#1731, Lunar and Planetary Institute, Houston, TX, March 1999.

[Feldman et al, 1998] W. C. Feldman, S. Maurice, A. B. Binder, B. L. Barraclough, R.C. Elphic, D. J. Lawrence, "Fluxes of Fast and Epithermal Neutrons from Lunar Prospector: Evidence for Water Ice at the Lunar Poles," Science, September 1998.

[Gennery, 1999] D. Gennery, "Traversability Analysis and Path Planning for a Planetary Rover", Autonomous Robots, 1999.

[Heiken et al, 1991] G. H. Heiken, D. T. Vaniman, B. M. French, eds., Lunar Sourcebook: a user's guide to the moon, Cambridge University Press, 1991.

[Ingersoll et al, 1992] A.P. Ingersoll, T. Svitek, B.C. Murray, "Stability of Polar Frosts in Spherical Bowl-Shaped Craters on the Moon, Mercury, and Mars", Icarus, Volume 100, pp. 40-47, 1992

[Johnson, 1979] N.L. Johnson, Handbook of Soviet Lunar and Planetary Exploration. Science and Technology Series, Volume 47, Amer. Astronau. Soc. Publ., 1979.

[Margot et al, 1999] J. L. Margot, R. F. Jurgens, M. A. Slade, "Locations of Cold Traps and Possible Ice Deposits Near the Lunar Poles: a Survey Based on Radar Topographic Mapping," in Lunar and Planetary Science XXX, abstract \#1897, Lunar and Planetary Institute, Houston, TX, March 1999.

[Schrunk, 1995] D. Schrunk, "Sun-Synchronous Operation", Workshop Discussion, ISE Lunar Conference, 1995. 